\title{
DIAGRAMAS DE PERFIL E DE COBERTURA DE UM TRECHO DA FLORESTA DE TABULEIRO NA RESERVA FLORESTAL DE LINHARES (ESPÍRITO SANTO, BRASIL) ${ }^{1}$
}

\author{
Ariane Luna Peixoto ${ }^{2,4}$ \\ Maria Mercedes Teixeira da Rosa ${ }^{2}$ \\ Luiz Carlos de Miranda Joels ${ }^{3}$
}

Recebido em 19.04.91. Aceito em 23.03.95.

\begin{abstract}
RESUMO: (Diagramas e perfil e de cobertura de um trecho da floresta de tabuleiro na Reserva Florestal de Linhares [Espírito Santo, Brasil]). O diagrama de perfil e de cobertura de um trecho de mata alta na floresta de tabuleiro da Reserva Florestal de Linhares, Espírito Santo, Brasil, foram obtidos a partir de duas amostras de $60 \mathrm{~m} \times 10 \mathrm{~m}$ e $30 \mathrm{~m} \times 20 \mathrm{~m}$, respectivamente, parcialmente superpostas. Todos os indivíduos com altura igual ou superior a $6 \mathrm{~m}$ e dap igual ou superior a $5 \mathrm{~cm}$ foram identificados e tiveram medidos a altura total, altura do fuste, dap e diâmetro da copa. Foram desenhados o fuste e as formas horizontal e vertical da copa. Os indivíduos foram classificados de acordo com o seu estádio de desenvolvimento em árvores potenciais, árvores do presente e árvores em decrepitude. Foram identificados três estratos com características próprias quanto às dimensões, composição florística e fase de desenvolvimento. Características estruturais da vegetação sucessora estão relacionadas com a maneira pela qual os indivíduos do dossel morrem, abrindo uma clareira ao cair, danificando indivíduos dos estratos inferiores ou perdendo lentamente partes vegetativas.
\end{abstract}

Palavras-chave: Hiléia bahiana, estratificação florestal, diagramas de perfil e cobertura, floresta atlântica.

\footnotetext{
ABSTRACT: (Profile and cover diagram of a section of the "tabuleiro" forest at the Linhares Forest Reserve; [Espírito Santo, Brasil]). The profile and the cover diagram of a section of the "tabuleiro" forest at the Linhares Forest Reserve, in the State of Espírito Santo, Brazil, were obtained from two samples of $60 \mathrm{~m} \times 10 \mathrm{~m}$ and $30 \mathrm{~m} \times 20 \mathrm{~m}$, respectively, partially overlapping. All trees in the sample which exceeded $6 \mathrm{~m}$ high and has $5 \mathrm{~cm}$ dbh or more were identified and measured for total height, trunk height, dbh and crown diameter. The trunk and the shapes of the crowns were

1 Trabalho realizado com auxílio do CNPq (Programa Linhas de Ação em Botânica, proc. 407871-86)

${ }^{2}$ Universidade Federal Rural do Rio de Janeiro, Instituto de Biologia, Caixa Postal 74582, 23851-970 Seropédica, Itaguaí, RJ, Brasil.

${ }^{3}$ Instituto Nacional de Pesquisas da Amazônia, Caixa Postal 478, 69011 Manaus, AM, Brasil.

${ }^{4}$ Pesquisador Bolsista do CNPq.
} 
drawn. The trees were classified according to their developmental degree into potential trees, present trees end decrepit trees. Three strata were found with distint dimensional, taxonomic and developmental features. Structural characteristic of the succeeding vegetation are related to the way trees in the upper stratum die, standing or falling.

Key words: Hiléia bahiana, Forest stratification, profile, diagram, atlantic forest.

\section{Introdução}

A região norte do Espírito Santo foi objeto de interesse de botânicos que, desde o início do século XIX empreenderam expedições científicas à área (Neuwied, 1820; Saint-Hilaire, 1818), descrevendo-a como uma das mais importantes regiões florestadas do sudeste brasileiro. Saint-Hilaire (1818), Aguirre (1950) e Egler (1954) escreveram sobre as formações arbóreas do norte do Espírito Santo, este último, destacando a pujança da mata de tabuleiro e o caráter de deciduidade sazonal de muitas espécies.

Os trabalhos de Heinsdijk et al. (1965), Borgonovi (1975) e Jesús (1982), desenvolvidos na Reserva Florestal de Linhares, iniciaram uma fase de estudos com ênfase florestal, tendo sido avaliado o potencial madeireiro, a qualidade da madeira e de outros produtos florestais da região norte do estado. Nos últimos anos, associados à inventários florísticos e faunísticos, os trabalhos passaram a ter ênfase notadamente ecológica e/ou conservacionista, refletindo a preocupação dos cientistas e da sociedade em geral com o processo acelerado de destruição dos ecossistemas naturais (Collar et al. 1987; Peixoto \& Peixoto 1986; Peixoto \& Gentry 1990).

A mata de tabuleiro no norte do Espírito Santo está associada a solos oligotróficos da Série Barreiras, com relevo plano e altitude entre 28 e $65 \mathrm{~m}$. A flora, rica e diversificada, apresenta várias espécies arbóreas endêmicas. Uma das formações arbóreas integrantes desta vegetação e a mais representativa encontrada na Reserva Florestal de Linhares $(63,10 \%$ da área total) é a mata alta, denominada por Jesús (1987), de floresta densa de cobertura uniforme. A mata alta, quando comparada com as outras formações integrantes da mata de tabuleiro, destaca-se por apresentar indivíduos de maior porte e sombreamento mais intenso do sub-bosque, que é ralo e facilmente penetrável. O conhecimento sobre a distribuição e a organização espacial das espécies arbóreas desse ecossistema poderá contribuir significativamente para a compreensão dos mecanismos ecológicos, permitindo inferências sobre a maturidade da comunidade e sobre as relações entre os indivíduos. Estas informações são importantes tanto para o conhecimento da floresta primitiva do norte do Espírito Santo, como para o desenvolvimento de atividades de manejo de ecossistemas florestais para fins silviculturais ou conservacionistas. Estudos fitossociológicos realizados anteriormente (Peixoto \& Gentry 1990) não caracterizaram nem discutiram a estratificação devido aos métodos empregados, que limitaram a discussão dos dados nesse aspecto.

Davis \& Richards (1933-1934), estudando a vegetação de um trecho de floresta na Guiana, utilizaram diagrama de perfil, visando a esclarecer controvérsias sobre a estratificação em floresta pluvial tropical. O método desenvolvido por aqueles autores foi posteriormente utilizado por Richards (1952) e Withmore (1975), entre outros, 
Diagramas de perfil e de cobertura da floresta da Reserva de Linhares, ES

sendo que este último o considera imprescindível para a caracterização das florestas tropicais.

O presente trabalho objetivou o estudo da estratificação e o estado de desenvolvimento das espécies ocorrentes nos diferentes estratos em um trecho de 0,1 ha da floresta alta na Reserva Florestal de Linhares.

\section{Material e métodos}

$\mathrm{O}$ trabalho foi efetuado em um trecho de mata alta adjacente à estrada X-2, próximo ao rio Barra Seca, numa área de $1.000 \mathrm{~m} 2$, subdividida em 5 lotes retangulares de $10 \mathrm{~m}$ x 20m, utilizando o método proposto por Davis \& Richards (1933-1934) e definido em termos de forma, tamanho e representação gráfica por Hallé et al. (1978). Para a elaboração do perfil delimitou-se uma faixa contínua de $60 \mathrm{~m} \times 10 \mathrm{~m}$ e para o diagrama de cobertura, uma faixa contínua de $20 \mathrm{~m}$ x $30 \mathrm{~m}$, sendo as áreas parcialmente coincidentes (Figura 1). O trecho foi cercado e subdividido com corda de náilon e não se procedeu a qualquer limpeza ou corte seletivo do sub-bosque. Foram incluídos todos os indivíduos de porte arbóreo com diâmetro do caule à altura do peito (dap) igual ou superior a $5 \mathrm{~cm}$. Consideraram-se apenas os indivíduos cujos troncos nasciam dentro da área analisada. Os espécimes que cresciam nos limites da área foram incluídos apenas quando a metade ou mais da base do tronco estava dentro da área estudada. De cada indivíduo foram anotadas medidas de: a) dap; b) altura total (Ht); c) altura do fuste (Hf), avaliadas quando possível com o auxílio do altímetro de Blume-Leiss, ou por homens de campo experientes em trabalhos de inventário florestal, cujo grau de precisão foi testado em amostragem ao acaso; d) diâmetro da copa, obtido pela distância no chão entre dois observadores colocados na projeção dos extremos da copa; e) desenho das formas horizontal e vertical da copa e do fuste. As árvores com sapopemas tiveram seu diâmetro medido acima do início do alargamento das sapopemas, quando este alargamento se dava acima de $1,30 \mathrm{~m}$. Nos espécimes cujos caules se ramificavam, o diâmetro de cada caule com dap maior que $5 \mathrm{~cm}$ foi registrado. Utilizou-se o cálculo da diferença Ht - Hf para a avaliação da altura da copa, sendo aí incluídas as primeiras ramificações que, a rigor, não fazem parte da copa.

De todos os indivíduos incluídos no estudo foram coletadas amostras para identificação. A coleta foi feita com o auxílio de tesoura de alta poda ou subindo-se nas árvores utilizando-se esporas. O material botânico foi processado e identificado nos herbários da Reserva Florestal de Linhares (CVRD) e do Departamento de Botânica da Universidade Federal Rural do Rio de Janeiro (RBR). Duplicatas de exemplares de diversos grupos taxonômicos foram enviadas a especialistas, quando necessário.

Para a avaliação do estádio de desenvolvimento dos indivíduos que ocorriam dentro da faixa estudada, analisaram-se 5 a 10 exemplares das mesmas espécies, ocorrentes fora da faixa estudada, anotando-se o dap, altura total, altura do fuste, forma da copa e outras observações, quando consideradas relevantes para o estudo. Diferenciaram-se os indivíduos em três grupos de maturidade, seguindo-se o postulado por Hallé et al. (1978) e Oldemann (1989); árvores potenciais, árvores do presente e árvo- 


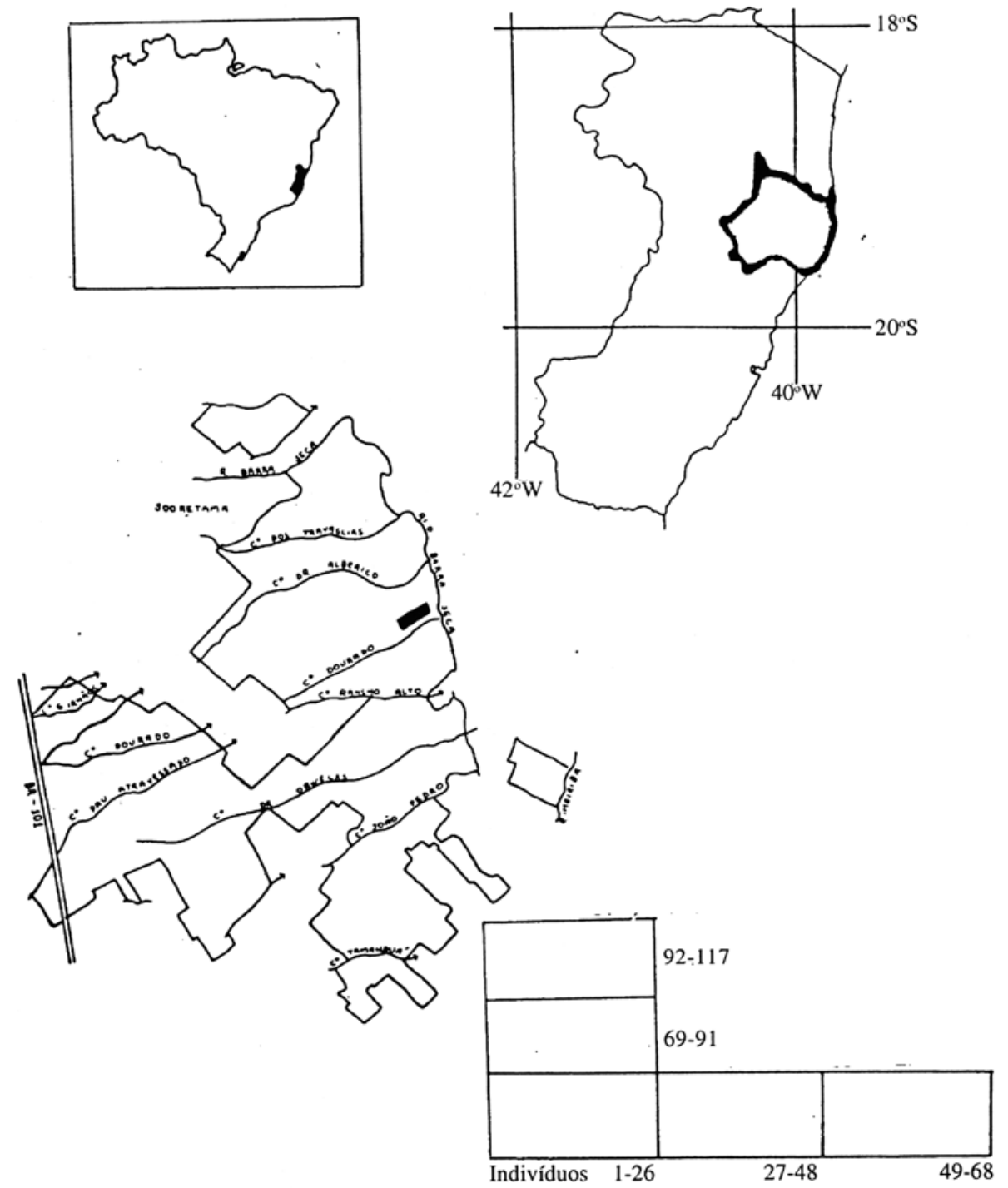

Figura 1 - Situação geográfica da Reserva Florestal de Linhares no estado do Espírito Santo e no Brasil, e croqui da área estudada.

res em decrepitude. Como árvores potenciais ou árvores do futuro consideraram-se aquelas com potencial para crescer em altura ou expansão (da copa e do fuste) ou ambos. Como árvores do presente consideraram-se aquelas cujo tamanho aparece como máximo ou próximo ao máximo, que têm capacidade para repor partes perdidas 
e parecem apresentar algum potencial para extensão ou expansão. Árvores em decrepitude ou árvores do passado são as que aparecem vetustas, decadentes, danificadas a um ponto que aparenta não ter recuperação ou que estão mortas.

Uma lista das espécies arbóreas da Reserva Florestal de Linhares encontra-se publicada em Jesús (1987).

Os dados climáticos foram obtidos na própria reserva florestal onde há um posto metereológico classe "A", sendo o diagrama climático elaborado com base em dados referente aos anos de 1982 a 1991 (Figura 2).

Reserva Florestal de Linhares (30m)

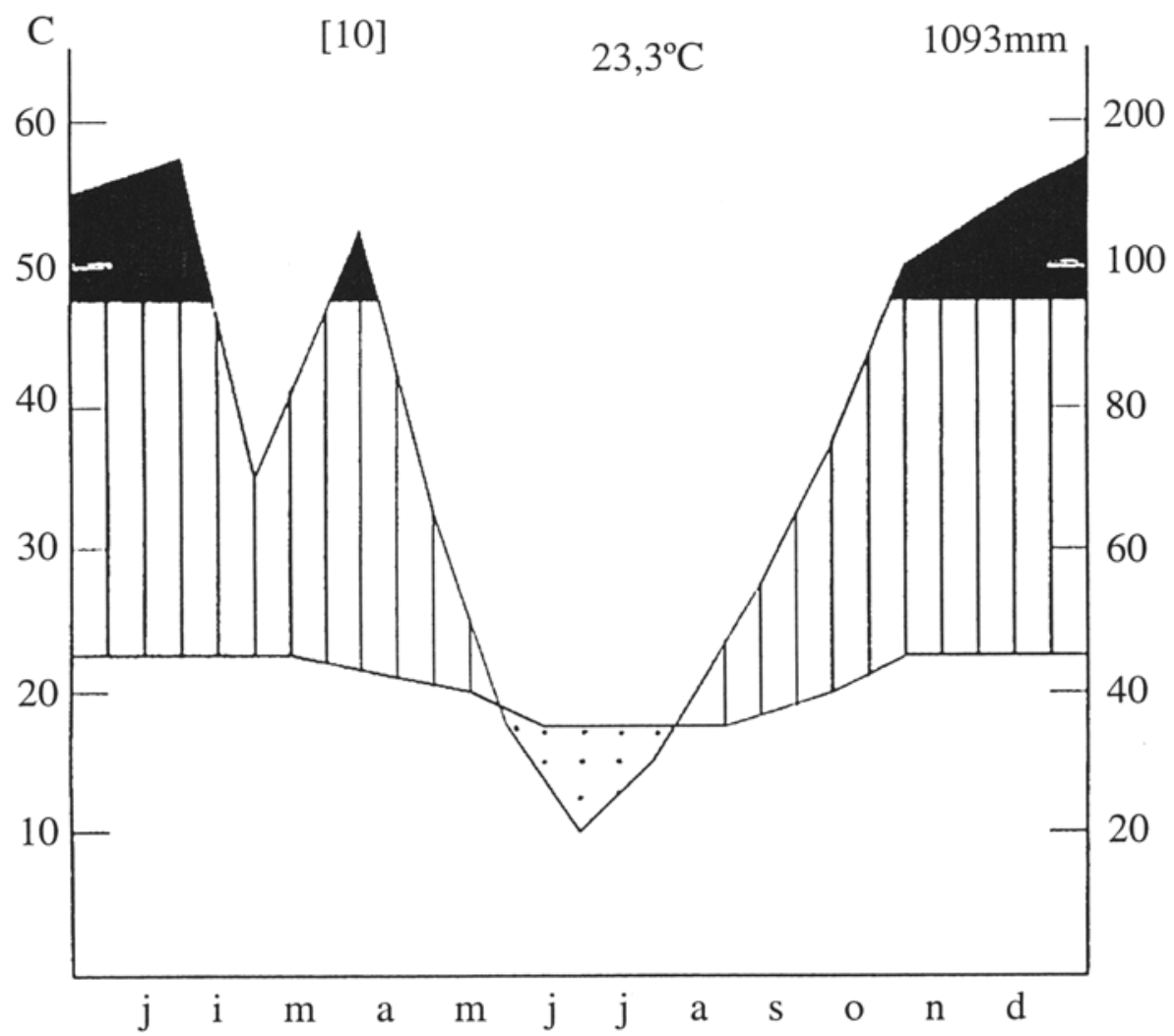

Figura 2 - Diagrama climático da Área da Reserva Florestal de Linhares, ES, com base em dados do período 1982-1991. 


\section{Resultados}

A área da Reserva Florestal de Linhares pode ser incluída na região climática Am de Köppen (1946). O clima é quente e úmido sendo a média anual de precipitação de $1.093 \mathrm{~mm}$, com uma estação seca, suave, de maio a setembro. O mês mais chuvoso é janeiro, com uma média de $187,3 \mathrm{~mm}$, sendo o valor máximo de 530,3mm (em 1985) e o valor mínimo de $2.8 \mathrm{~mm}$ em junho de 1985 . A média anual de temperatura é de 23,32 C, com mínima absoluta de 8,3 C em agosto de 1988 e a máxima absoluta de $39,8 \mathrm{C}$, em dezembro de 1990. A média anual de umidade relativa do ar é de $82.6 \%$ (Figura 2).

O solo Podzólico Vermelho-Amarelo distrófico, de textura média-argilosa, caracteriza-se por apresentar baixos teores de bases trocáveis $(\mathrm{Ca} 2+, \mathrm{MG} 2+\mathrm{e} \mathrm{K}+)$ e de fósforo e altos teores de alumínio trocável, indicando baixos índices de fertilidade natural (Peixoto \& Gentry 1990).

Em um trecho de 0,1 ha de floresta alta, na mata de tabuleiro, onde se empreendeu o estudo, foram amostrados 117 indivíduos com dap igual ou superior a $5 \mathrm{~cm}$, pertencentes a 68 espécies, 62 gêneros e 30 famílias (Tabela 1). A altura dos indivíduos oscilou entre $6 \mathrm{~m}$ e $31 \mathrm{~m}$, o diâmetro da copa entre $1 \mathrm{~m}$ e $14 \mathrm{~m}$ e o dap entre $5 \mathrm{~cm}$ e $90 \mathrm{~cm}$ (Tabela 2 e 3). Foram anotadas duas árvores de grande porte caídas mais ou menos recentemente, de modo que ainda se percebiam os efeitos de sua saída do sistema, duas árvores mortas em pé, com dap acima de $30 \mathrm{~cm}$, e 8 árvores mortas, com dap entre 5 e $15 \mathrm{~cm}$. Foi possível identificar visualmente três estratos arbóreos: estrato superior, estrato intermediário e estrato inferior.

Tabela 1 - Lista de espécimes em ordem de ocorrência na área de amostragem: dap = diâmetro à altura do peito; alt = altura total; fus = altura do fuste; $\operatorname{cop}=$ diâmetro da copa.

\begin{tabular}{|c|c|c|c|c|c|}
\hline Espécie & & $\begin{array}{c}\text { Família } \\
\text { dap } \\
\mathrm{cm}\end{array}$ & $\begin{array}{l}\text { alt } \\
\text { m }\end{array}$ & $\begin{array}{r}\text { fus } \\
\mathrm{m}\end{array}$ & $\begin{array}{r}\text { cop } \\
\text { m }\end{array}$ \\
\hline 1. Duguetia lanceolata St.Hil. & ANNONACEAE & 7 & 8 & 6 & 4 \\
\hline 2. Hydrogaster trinervis Kuhlm. & TILIACEAE & 90 & 30 & 19 & 12 \\
\hline 3. Myrcia racemosa (Berg)Kiaerk. & MYRTACEAE & 10 & 11 & 5 & $5 / 2.5$ \\
\hline 4. Astronium graveolens Jacq. & ANACARDIACEAE & 5 & 8 & 5 & $3.5 / 3$ \\
\hline 5. Trattinnickia burserifolia Mart & BURSERACEAE & 8 & 12 & 6 & 6 \\
\hline 6. Trichilia silvatica DC. & MELIACEAE & 8 & 10 & 5 & $5 / 4$ \\
\hline 7. Couepia schottii Fritsch & CHRYSOBALANACEAE & 17 & 18 & 13 & 5 \\
\hline 8. Schoepfia oblongifolia Turcz & OLACACEAE & 8 & 14 & 9 & 4 \\
\hline 9. Sterculia speciosa K.Schum. & STERCULIACEAE & 10 & 13 & 10 & 3 \\
\hline 10. Astrocaryum aculeatissimum (Schott)Burr. & PALMAE & 12 & 8 & 4 & 7 \\
\hline 11. Myrciaria amazonica Berg & MYRTACEAE & 6 & 10.2 & 6 & 2.5 \\
\hline 12. Myrcia racemosa (Berg)Kiaerk. & MYRTACEAE & 6 & 8 & 4.5 & 2 \\
\hline 13. Trichilia silvatica $\mathrm{DC}$. & MELIACEAE & 21 & 15 & 8 & 9 \\
\hline 14. Ocotea aciphylla (Nees)Mez & LAURACEAE & 5 & 8 & 6 & 2.5 \\
\hline 15. Senefeldera multiflora Mart. & EUPHORBIACEAE & 5 & 8.5 & 6.5 & 2.6 \\
\hline
\end{tabular}


Espécie

16. Franchetella sp.
17. Buchenavia hoehneana N.F.Mattos
18. Jacaratia heptaphylla (Vell.) A.DC.
19. Dialium divaricatum (Aubl.)Sandw.

20. Solanum alatirameum Bitter

21. Piptadenia adiantoides (Spreng.)Macbr.

22. Eriotheca candolleana (Schum.)Rob.

23. Enterolobium glaziovii (Benth.)Mesquita

24. Lecythis lanceolata Poiret

25. Trichilia silvatica DC.

26. Machaerium fulvovenosum Lima

27. Attalea humilis Mart.

28. Hydrogaster trinervis Kuhlm.

29. Tibouchina granulosa Camb.

30. Casearia decandra Jacq.

31. Franchetella sp.

32. Attalea humilis Mart.

33. Stephanopodium blanchetianum Baill.

34. Franchetella sp.

35. Spondias aff. purpurea $\mathrm{L}$.

36. Ecclinusa rafiflora Mart.

37. Alchornea triplinervea (Spreng.)M.Arg.

38. Dialium divaricatum (Aubl.)Sandw.

39. Geissospermum laeve (Vell.)Baill.

40. Centrolobium minus Presl.

41. Mouriri glazioviana Cogn.

42. Attalea humilis Mart.

43. Caesalpinia ferrea Benth.

44. Schoepfia oblongifolia Turcz

45. Dialium divaricatum (Aubl.)Sandw.

46. Pouteria pachycalyx Penn.

47. Eugenia aff. pyriflora Berg.

48. Tapirira sp.

49. Dialium divaricatum (Aubl.)Sandw.

50. Dialium divaricatum (Aubl.)Sandw.

51. Spondias aff. purpurea L.

52. Sorocea aff. guilleminiana Gaudich.

53. Spondias aff. purpurea L.

54. Simira grazielae A.L.Peixoto

55. Micropholis crassipedicellata (M.\& E.)Pier.

56. Ocotea divaricata (Poir.)Mez

57. Caesalpinia ferrea Benth.

58. Tachigalia multijuga Benth.

59. Pouteria pachycalyx Penn.

60. Dialium divaricatum (Aubl.)Sandw.

61. Marlierea gardneriana (Berg)Nied.

62. Tabebuia serratifolia Nich.

63. Enterolobium glaziovii (Benth.)Mesquita

\begin{tabular}{|c|c|c|c|c|}
\hline & $\begin{array}{c}\text { Família } \\
\text { dap } \\
\mathrm{cm}\end{array}$ & $\begin{array}{c}\text { alt } \\
\mathrm{m}\end{array}$ & $\begin{array}{r}\text { fus } \\
\mathrm{m}\end{array}$ & $\begin{array}{r}\text { cop } \\
\mathrm{m}\end{array}$ \\
\hline SAPOTACEAE & 5 & 8.5 & 6.5 & 2.6 \\
\hline COMBRETACEAE & 8 & 8 & 5 & 2.5 \\
\hline CARICACEAE & 30 & 18 & 10.5 & $7 / 5$ \\
\hline LEGUMINOSAE & 7 & 9 & 7 & 3.5 \\
\hline SOLANACEAE & 5 & 8 & 5 & 2.5 \\
\hline LEGUMINOSAE & 6 & 8 & 5 & 5 \\
\hline BOMBACACEAE & 5 & 8 & 6 & 3 \\
\hline LEGUMINOSAE & 33 & 31 & 23 & 14 \\
\hline LECYTHIDACEAE & 7 & 11 & 8 & 4 \\
\hline MELIACEAE & 8 & 8 & 5 & 4 \\
\hline LEGUMINOSAE & 75 & 31 & 22 & 12 \\
\hline PALMAE & 11.5 & 21 & 19 & 6 \\
\hline TILIACEAE & 29 & 19 & 11 & 5 \\
\hline MELASTOMATACEAE & 6.5 & 9 & 5 & 4 \\
\hline FLACOURTIACEAE & 7 & 8 & 5.5 & 4 \\
\hline SAPOTACEAE & 10.5 & 17 & 12 & 5 \\
\hline PALMAE & 10.5 & 9 & 8 & 5 \\
\hline DICHAPETALACEAE & 9 & 8.5 & 6 & 3 \\
\hline SAPOTACEAE & 18.5 & 14 & 9 & 6 \\
\hline ANACARDIACEAE & 21 & 15 & 12 & 5 \\
\hline SAPOTACEAE & 10 & 9 & 5.5 & 5.5 \\
\hline EUPHORBIACEAE & 11 & 11 & 9.5 & 2.5 \\
\hline LEGUMINOSAE & 20 & 13 & \multicolumn{2}{|c|}{$113.5 / 1.8$} \\
\hline APOCYNACEAE & 7 & 8 & 4 & 1.5 \\
\hline LEGUMINOSAE & 7.5 & 8 & 7 & 3.5 \\
\hline MELASTOMATACEAE & 6.5 & 7.5 & 6 & 2.5 \\
\hline PALMAE & 12 & 21 & 18 & 8 \\
\hline LEGUMINOSAE & 15 & 18 & 13 & 6 \\
\hline OLACACEAE & 10 & 8 & 6.5 & 2 \\
\hline LEGUMINOSAE & 34 & 26 & 18 & 12 \\
\hline SAPOTACEAE & 16.5 & 10 & 7 & 2 \\
\hline MYRTACEAE & 6.5 & 8 & 6 & 1.5 \\
\hline ANACARDIACEAE & 75 & 11 & 8 & 3 \\
\hline LEGUMINOSAE & 20 & 19 & 10 & $5 / 4$ \\
\hline LEGUMINOSAE & 39 & 21 & 4 & 12 \\
\hline ANACARDIACEAE & 8 & 9 & 6.5 & 1.2 \\
\hline MORACEAE & 8 & 9 & 6.5 & 1.2 \\
\hline ANACARDIACEAE & 66 & 31 & 21 & 13 \\
\hline RUBIACEAE & 5.5 & 9 & 7 & 1.2 \\
\hline SAPOTACEAE & 80 & 27.5 & 15 & 18 \\
\hline LAURACEAE & 11 & 14 & 11 & 2 \\
\hline LEGUMINOSAE & 5.5 & 12 & 8 & 1.5 \\
\hline LEGUMINOSAE & 9 & 18 & 11 & 4 \\
\hline SAPOTACEAE & 6 & 10 & 8 & 2 \\
\hline LEGUMINOSAE & 27.5 & 19 & 9.5 & 8 \\
\hline MYRTACEAE & 6.5 & 8 & 6 & 1.6 \\
\hline BIGNONIACEAE & 40 & 19 & 12 & 6 \\
\hline LEGUMINOSAE & 15 & 15 & 12.5 & 3 \\
\hline
\end{tabular}




\begin{tabular}{|c|c|c|c|c|c|}
\hline Espécie & & $\begin{array}{c}\text { Família } \\
\text { dap } \\
\mathrm{cm}\end{array}$ & $\begin{array}{c}\text { alt } \\
\mathrm{m}\end{array}$ & $\begin{array}{r}\text { fus } \\
\mathrm{m}\end{array}$ & $\begin{array}{r}\text { cop } \\
\mathrm{m} \\
\end{array}$ \\
\hline 64. Cariniana legalis (Mart.)O.ktze & LECYTHIDACEAE & 16.5 & 13 & 10 & 3 \\
\hline 65. Ocotea divaricata (Poir.)Mez & LAURACEAE & 7.5 & 9 & 6 & 3 \\
\hline 66. Tapirira sp. & ANACARDIACEAE & 7 & 10 & 6 & 2 \\
\hline 67. Caesalpinia ferrea Benth. & LEGUMINOSAE & 10 & 10 & 6.5 & 2 \\
\hline 68. Myrcia panicularis (Berg)N.Silveira & MYRTACEAE & 8 & 8 & 3.5 & 2 \\
\hline 69. Cathedra sp. & OLACACEAE & 23.5 & 23 & 19 & 7 \\
\hline 70. Myrcia gilsoniana Barroso \& Peixoto & MYRTACEAE & 7 & 7 & 4.5 & 2.5 \\
\hline 71. Alchornea triplinervea (Spreng.)M.Arg. & EUPHORBIACEAE & 6.5 & 10.5 & 7 & 3 \\
\hline 72. Licania arianeae Prance & CHRYSOBALANACEAE & 6 & 13 & 9 & 3 \\
\hline 73. Radlkoferella aff. venosa Mart. & SAPOTACEAE & 17 & 17 & 10 & 6.5 \\
\hline 74. Astronium graveolens Jacq. & ANACARDIACEAE & 42 & 28 & 22 & 7 \\
\hline 75. Schefflera morototoni (Aubl.)Mag.\&al. & ARALIACEAE & 14.5 & 15 & 8.5 & 4.5 \\
\hline 76. Senefeldera multiflora Mart. & EUPHORBIACEAE & 14 & 11 & 8 & 3 \\
\hline 77. Senefeldera multiflora Mart. & EUPHORBIACEAE & 5.5 & 9 & 7 & 2.5 \\
\hline 78. Byrsonima stipulacea A.Juss. & MALPIGHIACEAE & 14 & 17 & 13 & 4 \\
\hline 79. Neea sp. & NYCTAGINACEAE & 5 & 9 & 5 & $1 / 2$ \\
\hline 80. Eugenia stictosepala Kiaerk. & MYRTACEAE & 18.5 & 15.5 & 9 & 3.2 \\
\hline 81. Eugenia tinguiyensis Camb. & MYRTACEAE & 10 & 13 & 8 & 3 \\
\hline 82. Trattinnickia burserifolia Mart. & BURSERACEAE & 10.6 & 16 & 12 & 5.5 \\
\hline 83. Pourouma cinerascens Mart. ex Miq. & MORACEAE & 16.5 & 10.5 & 7 & 2.5 \\
\hline 84. Eugenia oxyphylla Berg. & MYRTACEAE & 7 & 13.5 & 8 & 3 \\
\hline 85. Eriotheca candolleana (Schum.)Rob. & BOMBACACEAE & 17 & 16 & 12 & 5 \\
\hline 86. Euterpe edulis Mart. & PALMAE & 6 & 6 & 4 & 5 \\
\hline 87. Manilkara salzmanii (A.DC.)Lam. & SAPOTACEAE & 22.3 & 18 & 14 & 5 \\
\hline 88. Manilkara salzmanii (A.DC.)Lam. & SAPOTACEAE & 7.4 & 12.5 & 8 & 3 \\
\hline 89. Helicostilis tomentosa (Poepp.\& Endl.)Rusby & MORACEAE & 12 & 13 & 9 & 2.7 \\
\hline 90. Sorocea aff. guilleminiana Gaudich. & MORACEAE & 7.5 & 8 & 4 & 2.8 \\
\hline 91. Protium macrophyllum Engl. & BURSERACEAE & 39.5 & 23.5 & 19 & 4 \\
\hline 92. Franchetella sp. & SAPOTACEAE & 6 & 9.5 & 5 & 7 \\
\hline 93. Dialium divaricatum (Aubl.)Sandw. & LEGUMINOSAE & 12 & 14 & 8 & $4 / 3$ \\
\hline 94. Aspidosperma olivaceum M.Arg. & APOCYNACEAE & 9 & 14 & 8 & 6 \\
\hline 95. Sorocea aff. guilleminiana Gaudich. & MORACEAE & 11 & 11.5 & 9 & 5 \\
\hline 96. Alchornea triplinervea (Spreng.)M.Arg. & EUPHORBIACEAE & 19 & 17 & 12 & 6 \\
\hline 97. Trichilia silvatica DC. & MELIACEAE & 6.5 & 8.2 & 5 & 6 \\
\hline 98. Franchetella sp. & SAPOTACEAE & 12 & 12 & 7 & 4 \\
\hline 99. Astrocaryum aculeatissimum (Schott)Burr. & PALMAE & 11 & 11 & 8 & 6 \\
\hline 100. Myrcia gilsoniana Barroso \& Peixoto & MYRTACEAE & 5.5 & 8.3 & 6 & 2 \\
\hline 101. Radlkoferella aff. venosa Mart. & SAPOTACEAE & 16 & 14.3 & 5.5 & 6 \\
\hline 102. Joannesia princeps Vell. & EUPHORBIACEAE & 18 & 19 & 14 & 4 \\
\hline 103. Swartzia flaemingii Raddi & LEGUMINOSAE & 8.5 & 9.5 & 3.5 & 3.3 \\
\hline 104. Cordia sellowiana Cham. & BORAGINACEAE & 6 & 14 & 9 & 1 \\
\hline 105. Tachigalia multijuga Benth. & LEGUMINOSAE & 15.5 & 13 & 11 & 3 \\
\hline 106. Eschweilera ovata (Camb.)Miers & LECYTHIDACEAE & 18 & 14.5 & 7 & 5.5 \\
\hline 107. Trichilia silvatica DC. & MELIACEAE & 32 & 20 & 15 & 11.5 \\
\hline 108. Inga nuda Salsz. & LEGUMINOSAE & 7.5 & 8.5 & 5.5 & 2.3 \\
\hline 109. Marlierea gardneriana (Berg)Nied. & MYRTACEAE & 6 & 8.5 & 4 & 1 \\
\hline 110. Vataireopsis araroba (Aguiar)Ducke & LEGUMINOSAE & 22 & 19 & 14 & 6 \\
\hline 111. Franchetella sp. & SAPOTACEAE & 27 & 9 & 6 & 3 \\
\hline
\end{tabular}




\begin{tabular}{lrrrrr} 
Espécie & & Família & & \\
dap & alt & fus \\
cm & m & $\begin{array}{r}\text { cop } \\
\text { m }\end{array}$ \\
\hline 112. Tabebuia serratifolia Nich. & BIGNONIACEAE & 59 & 26 & 20 & 5.6 \\
113. Astronium graveolens Jacq. & ANACARDIACEAE & 58 & 30 & 24 & 10.5 \\
114. Couepia schotii Fritsch & CHRYSOBALANACEAE & 11.5 & 15.5 & 11.5 & 3 \\
115. Jacaratia heptaplyylla (Vell.) A.DC. & CARICACEAE & 13 & 13 & 10 & 3 \\
116. Aspidosperma olivaceum M.Arg. & APOCYNACEAE & 10 & 10 & 5 & 5 \\
117. Ocotea aciphylla (Nees)Mez & LAURACEAE & 9 & 9.2 & 6 & 2 \\
\hline
\end{tabular}

Tabela 2. Número de indivíduos e táxons amostrados.

\begin{tabular}{lcccc}
\hline & espécimes & espécies & gêneros & famílias \\
\hline estrato superior & 21 & 14 & 14 & 10 \\
estrato intermediário & 46 & 34 & 32 & 20 \\
estrato inferior & 50 & 42 & 38 & 21 \\
\hline
\end{tabular}

Tabela 3. Dados médios, mínimos e máximos do grupamento de espécies dos estratos.

\begin{tabular}{|c|c|c|c|}
\hline & $\begin{array}{r}\text { estrato } \\
\text { superior }\end{array}$ & $\begin{array}{r}\text { estrato } \\
\text { intermediário } \\
\end{array}$ & $\begin{array}{l}\text { estrato } \\
\text { inferior }\end{array}$ \\
\hline $\begin{array}{l}\text { Altura máxima } \\
(\mathrm{m})\end{array}$ & 31 & 18 & 10.5 \\
\hline $\begin{array}{l}\text { Altura mínima } \\
(\mathrm{m})\end{array}$ & 19 & 11 & 6 \\
\hline $\begin{array}{l}\text { Altura média } \\
(\mathrm{m})\end{array}$ & $\begin{array}{c}24 \\
(24.32)\end{array}$ & $\begin{array}{c}14.13 \\
(14.21)\end{array}$ & $\begin{array}{c}8.68 \\
(8.73)\end{array}$ \\
\hline $\begin{array}{l}\text { dap máximo } \\
(\mathrm{cm})\end{array}$ & 90 & 75 & 27 \\
\hline $\begin{array}{l}\text { dap mínimo } \\
(\mathrm{cm})\end{array}$ & 18 & 5.5 & 5 \\
\hline dap médio & 40.52 & 14.57 & 7.89 \\
\hline$(\mathrm{cm})$ & $(43.55)$ & $(14.65)$ & (7.88) \\
\hline $\begin{array}{l}\text { cop máximo } \\
(\mathrm{m})\end{array}$ & 14 & 9 & 7 \\
\hline $\begin{array}{l}\text { cop mínimo } \\
\text { (m) }\end{array}$ & 4 & 1 & 1 \\
\hline cop médio & 8.69 & 5.06 & 3.25 \\
\hline (m) & $(8.87)$ & $(5.04)$ & (3.09) \\
\hline Ht-Hf médio & 7.02 & 4.66 & 3.02 \\
\hline (m) & $(7.5)$ & $(4.68)$ & (3.06) \\
\hline Relação Ht-Hf/ & 29.25 & 33.47 & 35.02 \\
\hline
\end{tabular}

O estrato superior, compreendendo as árvores com alturas entre $19 \mathrm{~m}$ e $31 \mathrm{~m}$, é descontínuo e tem altura média de $24 \mathrm{~m}$. As maiores alturas estão representadas por indivíduos de Enterolobium glaziovii $(31 \mathrm{~m})$, Machaerium fulvovenosum $(31 \mathrm{~m})$, Spon- 
dias aff. purpurea $(31 \mathrm{~m})$, Hydrogaster trinervis $(30 \mathrm{~m})$ e Astronium graveolens $(30 \mathrm{~m})$. $\mathrm{Na}$ área amostrada não há indivíduos emergentes, embora sejam relativamente comuns em outros trechos da mata. Os dap das árvores desse estrato oscilam entre $18 \mathrm{~cm}$ e $90 \mathrm{~cm}$ (excetuando Attalea humilis), sendo o dap médio $40,52 \mathrm{~cm}$. Os indivíduos desse estrato apresentam copas amplas que variam entre $4 \mathrm{~m}$ e $14 \mathrm{~m}$ de diâmetro, sendo a diferença Ht-Hf média de 7,02m, o que representa $29,25 \%$ da altura média total (Tabela 3).

No estrato superior predominam árvores do presente. Dos indivíduos amostrados, 63, $1 \%$ encontram-se nesse grupo, estando representados por espécimes de Joannesia princeps, Protium macrophyllum, Micropholis crassipedicellata, Hydrogaster trinervis, Tabebuia serratifolia, Astronium graveolens, Dialium divaricatum, Trichilia silvatica, Enterolobium glaziovii, Spondias aff. purpurea. Das 5 últimas espécies foram encontradas árvores potenciais no estrato intermediário e/ou inferior. Árvores em decrepitude contribuem nesse estrato com $26,3 \%$, estando representadas por espécimes de Tabebuia serratifolia, Cathedra sp., Dialium divaricatum e Astronium graveolens. Como árvores potenciais foram encontrados dois espécimes de Vataireopsis araroba e Hydrogaster trinervis, que contribuem com 10,5\% no total de árvores do estrato superior.

O estrato intermediário está constituído por árvores de $11 \mathrm{~m}$ a $18 \mathrm{~m}$, com altura média de 14,13m. Os espécimes com maior altura são de Manilkara salzmanii (18m), Jacaratia heptaphylla $(18 \mathrm{~m})$, Tachigalia multijuga $(18 \mathrm{~m})$, Caesalpinia ferrea $(18 \mathrm{~m})$ e Couepia schotii $(18 \mathrm{~m})$. Os dap das árvores desse estrato oscilam entre $5,5 \mathrm{~cm} \mathrm{e}$ $75 \mathrm{~cm}$, sendo o dap médio de $14,57 \mathrm{~cm}$. Os diâmetros das copas variam entre $1 \mathrm{~m}$ e $9 \mathrm{~m}$, sendo a diferença média $\mathrm{Ht}-\mathrm{Hf}$ de $4,73 \mathrm{~cm}$, o que representa $33,47 \%$ da altura média total (Tabela 3).

No estrato intermediário predominam indivíduos em crescimento (árvores potenciais), que contribuem com $52,17 \%$. Árvores do presente constituem $41,30 \%$ das árvores desse estrato, estando representadas por Alchornea triplinervea, Sorocea guilleminiana, Tratinnickia burserifolia, Jacaratia heptaphylla, Tachigalia multijuga, Licania arianeae, Schoepfia oblongifolia, Cordia sellowiana, Eugenia stictosepala, Franchetella sp., Byrsonima stipulacea, Radlkoferella aff. venosa, Couepia schotii, Caesalpinia ferrea e Manilkara salzmanii. Árvores em decrepitude representam 6,52\% desse estrato, fazendo parte desse contingente Ocotea divaricata, Myrcia racemosa e Eugenia tinguiyensis.

O estrato inferior, com árvores entre $6 \mathrm{~m}$ e $10,5 \mathrm{~m}$ de altura, tem altura média de $8,68 \mathrm{~m}$. Os espécimes com maior altura são Pourouma cinerascens $(10,5 \mathrm{~m})$, Alchornea triplinervea $(10,5 \mathrm{~m})$, Caesalpinia ferrea $(10 \mathrm{~m})$, Tapirira $\mathrm{sp} .(10 \mathrm{~m})$, Pouteria pachycalyx $(10 \mathrm{~m})$, Aspidosperma olivaceum $(10 \mathrm{~m})$, Myrciaria amazonica $(10,2 \mathrm{~m}) \mathrm{e}$ Trichilia silvatica $(10 \mathrm{~m})$. Os dap das árvores desse estrato oscilam entre 5 e $27 \mathrm{~cm}$, sendo o dap médio de $7,8 \mathrm{~cm}$. Os diâmetros das copas variam entre $1 \mathrm{~m}$ e $7 \mathrm{~m}$, sendo a diferença $\mathrm{Ht}-\mathrm{Hf}$ média de $3,04 \mathrm{~m}$ o que representa $35,02 \%$ da altura total (Tabela 3).

No estrato inferior predominam indivíduos jovens de espécies dos estratos intermediário e superior (76,59\%). Como árvores do presente, $21,27 \%$ desse estrato, 
Diagramas de perfil e de cobertura da floresta da Reserva de Linhares, ES

foram encontrados Myrcia racemosa, Solanum alatirameum, Eugenia pyriflora, Myrcia gilsoniana, Marlierea gardneriana, Inga nuda, Neea sp., Tibouchina granulosa, Casearia decandra, Swartzia flaemingii, sendo que as três últimas podem aparecer como árvores do presente, também, no estrato intermediário, de acordo com os dados obtidos em indivíduos localizados fora da área de amostragem. As árvores em decrepitude representam apenas $2,12 \%$ do total e correspondem a um indivíduo de Pouteria pachycalyx, que não alcançou seu desenvolvimento completo, ramificou-se, adquiriu posição inclinada e encontra-se decrepito. Não foi possível definir com segurança se a decrepitude foi motivada por um agente externo, biótico ou abiótico, ou por deficiência do próprio indivíduo.

\section{Discussão}

O Método - Hallé et al. (1978) afirmaram que, de modo geral, a dimensão para o perfil de um lote deve ter a largura entre $1 / 3$ e 2/3 da altura da floresta e o comprimento deve ser no mínimo igual a sua altura. Em florestas tropicais com árvores de altura superior a $30 \mathrm{~m}$ o perfil não deve ser muito grande, de modo a minimizar o risco da introduzir distúrbios de diferentes origens.

Oldemann (1974), em florestas na Guiana Francesa, usou parcelas de 20mx30m e considerou apenas as árvores com altura superior a $10 \mathrm{~m}$. Com esse procedimento elaborou diagramas de perfil fiéis e legíveis. Na Reserva Florestal de Linhares, usando-se o limite de 10m de altura utilizado por Oldemann (1974), seriam excluídos em 0, 1ha amostrado 41 indivíduos, dentre os quais um importante contingente de espécies típicas do estrato inferior, principalmente da família Myrtaceae. Esta família é reportada como a mais rica em número de espécies nas florestas do norte do Espírito Santo (Peixoto \& Gentry 1990) e Sul da Bahia (Mori et al. 1983). No estrato inferior as Myrtaceae estão representadas por 8 indivíduos, pertencentes a 7 espécies, das quais apenas 2 podem aparecer como integrantes do estrato intermediário (Myrcia racemosa e Eugenia tinguiyensis). Ainda nessa família, Eugenia stictosepala alcança sua maturidade no estrato intermediário e Eugenia oxyphylla, que aparece como árvore potencial, atinge sua maturidade no estrato superior, quando pode alcançar $28 \mathrm{~m}$ de altura (dados coligidos em exemplares de fora da área amostral).

Richards (1952) sugeriu a prévia limpeza da área a ser estudada, com a retirada dos indivíduos de até $7 \mathrm{~m}$ de altura. Na floresta alta, em Linhares, esse procedimento tornou-se desnecessário devido à escasez de vegetação no sub-bosque, que permitiu a execução do trabalho sem a retirada de qualquer indivíduo.

A estratificação horizontal - Rollet (1974) discutiu amplamente os diversos conceitos de estratificação florestal, sendo alguns dos seus postulados sumarizados por Hallé et al. (1974). No presente trabalho a estratificação é entendida não como uma subdivisão estática do conjunto de populações da floresta, mas como o grupamento de árvores que, em um determinado momento, atingem em conjunto, uma organização horizontal perceptível. A esse conjunto horizontal podem pertencer de forma transitória árvores que, se alcançarem seu desenvolvimento pleno, integarão estratos 
superiores. A concentração das copas das árvores em determinado espaço, constituindo estratos mais ou menos definidos foi analisada por Smith (1973), que reconheceu a implicação de muitos fatores bióticos ligados à fisiologia das árvores, ao modelo arquitetural de crescimento e a interrelação com animais polinizadores, dispersores, predadores e outros.

Embora Grubb et al. (1963) afirmassem que não há, em nenhuma parte do mundo, evidência da existência de três estratos constituídos por espécies arbóreas com descontinuidade bem marcada entre eles e que o reconhecimento dos estratos numa população lenhosa procede de um julgamento subjetivo, habitualmente em florestas tropicais úmidas são reconhecidos três estratos arbóreos. A localização desses estratos e a sua estruturação de maneira mais ou menos contínua varia nas diferentes áreas estudadas.

Três estratos arbóreos foram reconhecidos em uma floresta densa de montanha, em Bornéu (Richards 1936), com níveis superiores a 8m, 18m e 34m; também em uma floresta ao sul da Nigéria (Richards 1939), aos níveis de $15 \mathrm{~m}, 37 \mathrm{~m}$ e mais de $46 \mathrm{~m}$. Schulz (1960), em uma floresta no Suriname, identificou três estratos arbóreos, com níveis superiores a $18 \mathrm{~m}, 28 \mathrm{~m}$ e $45 \mathrm{~m}$. Silva (1980) num trecho de mata atlântica no município de Ubatuba, estado de São Paulo, identificou três estratos arbóreos, ditos descontínuos, com níveis superiores a $13 \mathrm{~m}, 24 \mathrm{~m}$ e $32 \mathrm{~m}$. Na Reserva Florestal de Linhares, no trecho de mata alta estudado, identificaram-se três estratos arbóreos, o inferior e o intermediário com copas bem entrelaçadas e o superior com acentuada descontinuidade entre as copas de seus constituintes.

$\mathrm{Na}$ área estudada o contingente mais abundante do estrato inferior é aquele constituído por indivíduos em crescimento de espécies dos estratos seguintes, enquanto no estrato superior o contingente mais representativo é aquele de indivíduos com maturidade plena ou quase. No total o número de indivíduos em crescimento na área estudada é maior do que o de maduros, o que demonstra que muitos daqueles que têm potencial para alcançar os estratos superiores podem, por motivos diversos, não os atingir. A decrepitude e a morte atingem diferentes indivíduos independentemente do porte e do estrato que integrem. Esse fato, citado por Swaine (1989), pode ser claramente percebido na área estudada pela presença de 8 espécimes mortos com dap entre $5 \mathrm{~cm}$ e $15 \mathrm{~cm}$ além de 4 espécimes de grande porte, dois dos quais ainda em pé. Os integrantes dos estratos inferiores, após a morte, são especialmente difíceis de caracterizar pela impossibilidade de avaliar a altura, proceder à identificação das espécias e pelo fato de se deteriorarem, de maneira geral, de modo mais rápido.

O processo e a dinâmica de crescimento da floresta podem ser diagnosticados pelo estado dos componentes arbóreos. $\mathrm{Na}$ área onde se localizam os indivíduos 1 a 68 (Figuras 3 e 4) há um trecho no qual a estratificação, nesse momento, não está definida. Esse trecho, compreendido entre os indivíduos 35 e 41, sofreu o distúrbio causado pela queda de uma árvore que se encontra em início de decomposição sobre o solo, percebendo-se aí os efeitos causados pela sua saída do sistema como planta viva, através da entrada de maior luminosidade para os estratos inferiores. Os indivíduos desse trecho encontram-se todos em crescimento, como árvores do futuro, exce- 
to Alchornea triplinervea (indivíduo 37) que é uma árvore do presente. A quantidade de indivíduos não incluídos na amostragem por apresentar dap menor que $5 \mathrm{~cm}$ é grande, nesse trecho.

Subparcelas verticais - Entendendo-se a floresta como um mosaico de diferentes tamanhos e em diferentes estádios de desenvolvimento (Oldeman, 1989), o trecho analisado mostra-se constituído de subparcelas (eco-unidades) mais ou menos densas e com diferentes níveis de superposição e entrelaçamento da massa foliar, o que confere à estratificação horizontal certa descontinuidade, especialmente nos estratos superiores. Assim, na Figura 3, a subparcela na qual estão localizados os indivíduos 1 a 26 e 42 a 60 é densa e com vários níveis de cobertura, enquanto o trecho no qual estão os indivíduos 35 a 41 é pouco denso em indivíduos amostrados e o entrelaçamento da massa foliar é menor e sem uma organização definida. Nesse trecho, nesse momento, não estão definidas as árvores que o comporão, não sendo possível caracterizá-lo como eco-unidade florestal madura. Os indivíduos, tanto os amostrados como os que estavam abaixo do padrão da amostragem encontram-se agregados de forma desorganizada.

Em algumas subparcelas, a superposição das copas chega a 4 níveis, mais raro a 5 níveis, como entre os indivíduos 1 a 15 (Figura 3 e 4). Através da sobreposição das copas, do entrelaçamento da massa foliar e do estádio de desenvolvimento dos componentes observa-se que as eco-unidades compreendidas pelos indivíduos 1 a 15, 42 a 58 e 92 a 107 (Figura 4) parecem representar modelos de grupamento florestal em homeostase.

Sem levar em conta as implicações genéticas, considerando apenas o estádio de desenvolvimento das árvores no momento da amostragem e as relações entre os indivíduos componentes da área estudada, observa-se que diferentes indivíduos de uma mesma espécie podem ter padrões de crescimento distintos. Analisando-se os indivíduos 57 e 67 de Caesalpinia ferrea, ilustrados no perfil da Figura 3, observa-se que o espécime com $12 \mathrm{~m}$ de altura tem dap de $5,5 \mathrm{~cm}$ e diâmetro da copa de $1,5 \mathrm{~m}$, enquanto o espécime com $10 \mathrm{~m}$ de altura tem dap de $10 \mathrm{~cm}$, diâmetro da copa de $2 \mathrm{~m}$. O exemplar 67 tem apenas dois níveis de cobertura acima de sua copa, enquanto o exemplar 57 tem 4 níveis de cobertura. Embora na área amostrada Caesalpinia ferrea esteja representada por indivíduos potenciais, foram feitas observações em cinco árvores consideradas do presente, das quais se anotaram os seguintes dados dap $=132 \mathrm{~cm}, 98 \mathrm{~cm}$, $55 \mathrm{~cm}, 52 \mathrm{~cm}, 44 \mathrm{~cm}$; altura total $=25 \mathrm{~m}, 32 \mathrm{~m}, 25 \mathrm{~m}, 27 \mathrm{~m}, 26 \mathrm{~m}$; altura do fuste $=12 \mathrm{~m}$, $20 \mathrm{~m}, 16 \mathrm{~m}, 18 \mathrm{~m}, 16 \mathrm{~m}$; diâmetro da copa $=22 \mathrm{~m}, 23 \mathrm{~m}, 10 \mathrm{~m}, 22 \mathrm{~m}, 18 \mathrm{~m}$.

A presença de espécies de porte arbóreo da família Palmae, confere às florestas tropicais características fisionômicas peculiares. Na área estudada foram amostradas 3 espécies de Palmae: Euterpe edulis, com 1 espécime no estrato inferior, Astrocaryum aculeatissimum, com 2 espécimes integrando o estrato inferior e Attalea humilis, com 3 espécimes alcançando o estrato superior. Silva (1980) em duas transeções de $75 \mathrm{~cm} \times 10 \mathrm{~cm}$, feitas num trecho de mata atlântica encontrou 6 espécimes de Astrocaryum aculeatissimum no estrato inferior, em cada uma das transeções, e Euterpe edulis com 7 indivíduos em uma transeção e 6 em outra, chegando ao estrato superior. 

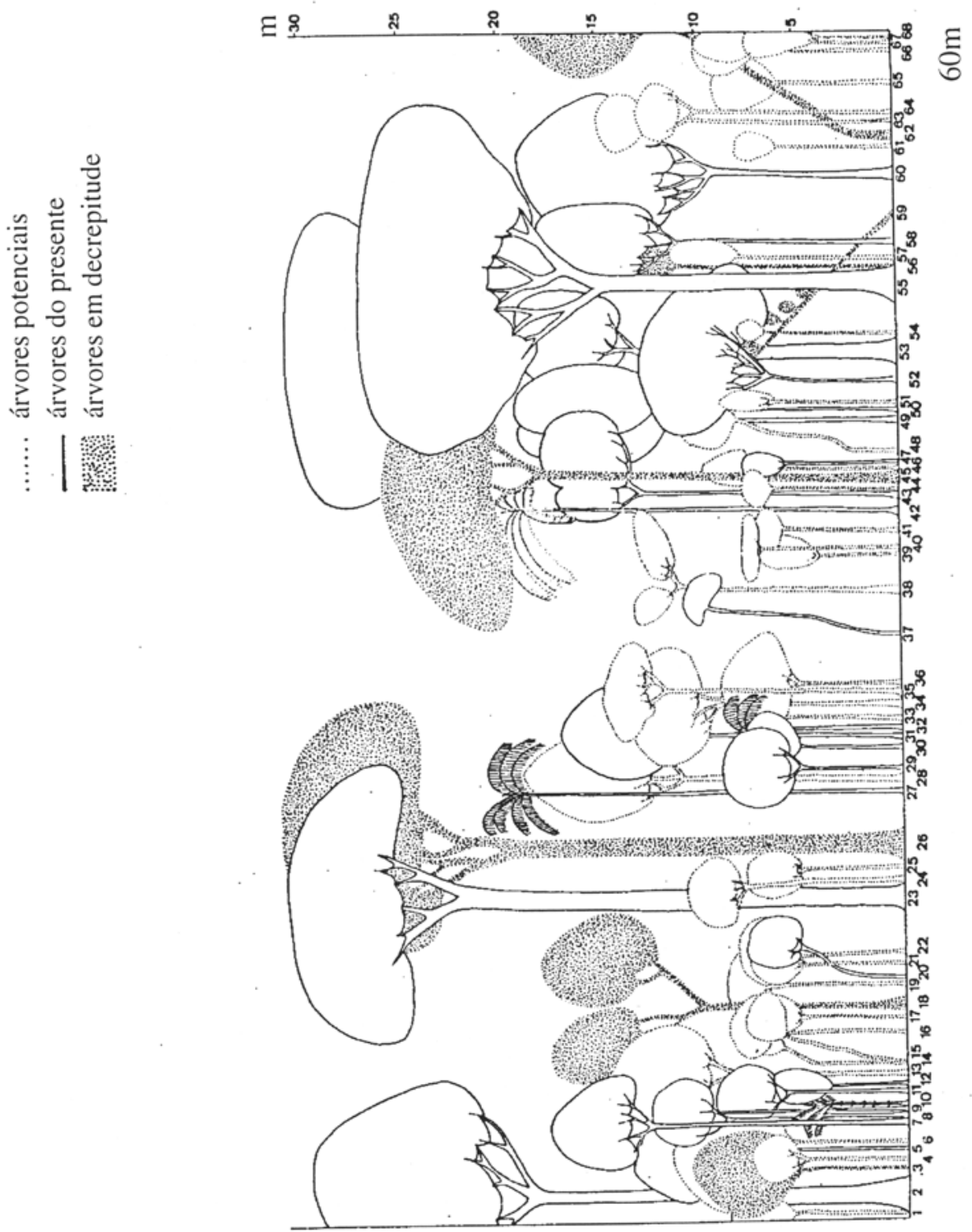

Figura 3 - Diagrama de perfil em transeções de $60 \mathrm{~m}$ x 10m. Os números correspondem aos dos espécimes listados na tabela 1 . 


\section{......... árvores potenciais \\ - árvores do presente \\ ----- árvores em decrepitude}

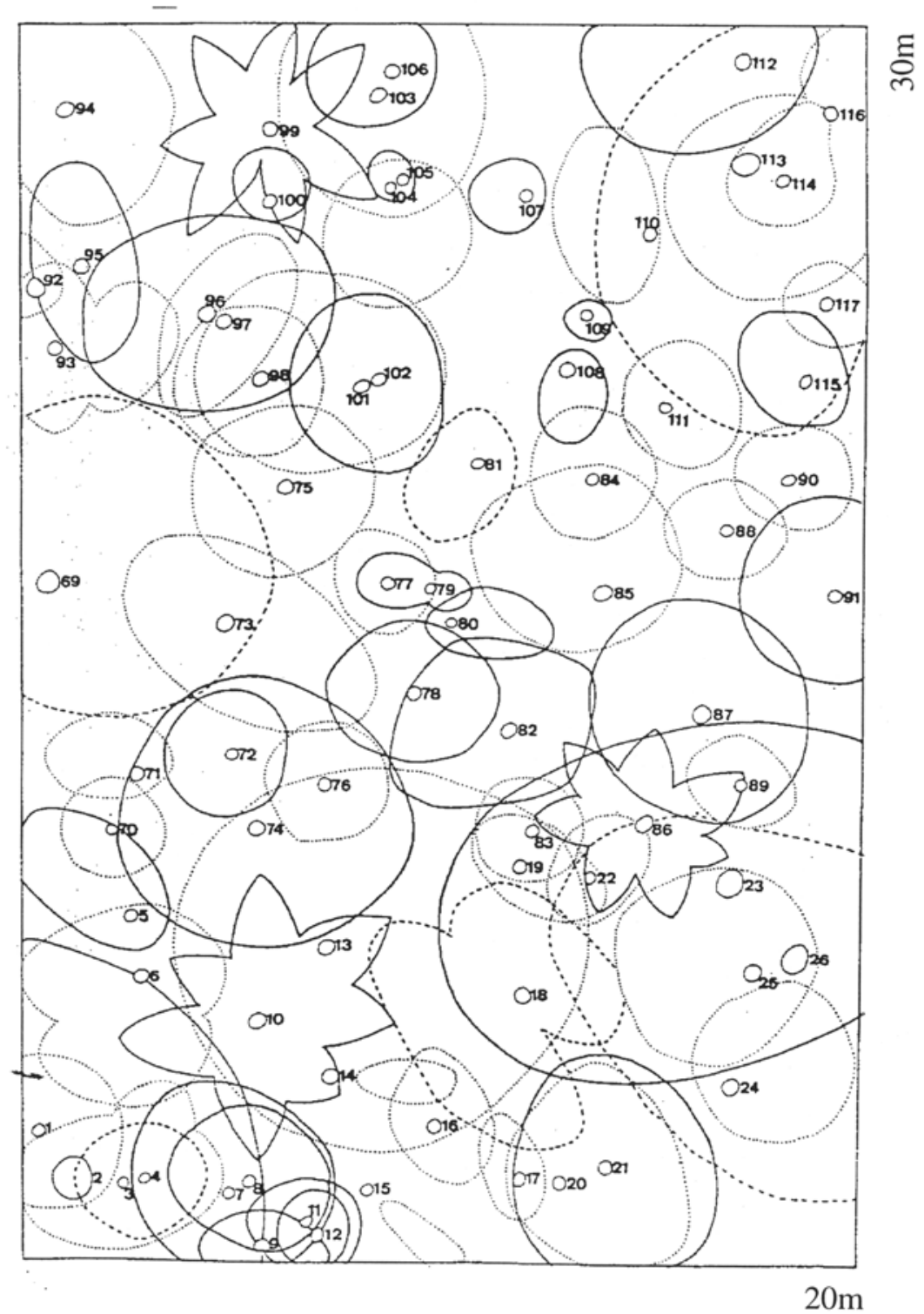

Figura 4 - Diagrama de cobertura em transeções de $20 \mathrm{~m}$ x 30m. Os números correspondem aos dos espécimes listados na tabela 1. 
$\mathrm{Na}$ área estudada foram encontrados 2 indivíduos de grande porte, com dap superior a $30 \mathrm{~cm}$, mortos, porém com seus troncos em decomposição ainda em pé. O processo de decrepitude pode ser percebido pela queda de galhos de diferentes tamanhos durante um período aparentemente longo de tempo. As eco-unidades nas quais essas árvores mortas se encontravam aparentemente não sofreram impacto com a saída desses indivíduos do sistema como planta viva.

\section{Conclusões}

A fisionomia florestal pode ser demonstrada, além de descrita, através de ilustrações de diagramas de perfil e de cobertura.

A caracterização fisionômica de trechos florestais, quando associada a dados numéricos e da estrutura de populações de espécies, dá imagem da heterogeneidade e diversidade da floresta, fornecendo meios para a elaboração de modelos ecológicos.

Os três estratos arbóreos identificados na mata alta da Reserva Florestal de Linhares têm características próprias quanto à composição florística, dimensões e estádio de desenvolvimento de seus componentes. No estrato superior, sem um dossel contínuo, predominam árvores do presente, enquanto no estrato inferior, com massa foliar densa e entrelaçada, predominam árvores do futuro, representadas, em sua maioria, por indivíduos jovens de espécies que poderão chegar aos estratos superiores.

$\mathrm{Na}$ área estudada, a saída de árvores pode provocar distúrbios no dossel florestal, provocando alterações nos estratos inferiores, desorganizando o sistema e impossibilitando a visão da estratificação florestal. Entretanto, quando a saída de indivíduos do sistema ocorre ao longo de um período de tempo maior, com a perda sucessiva de galhos e do fuste, não causa distúrbio aparente na estratificação, sendo o processo morte/renovação feito de maneira mais lenta, não havendo a formação aparente de uma clareira.

A morte e a renovação ocorrem independentemente do tamanho dos indivíduos, em cada estrato. Entretanto, no estrato inferior é mais difícil perceber a morte e a saída de seus integrantes do sistema vivo.

É difícil reconhecer o estádio de desenvolvimento de espécies arbóreas sem que se examinem diversos exemplares aparentemente em maturidade plena.

\section{Agradecimentos}

Ao Engo. Florestal M. de S. Menandro, da Reserva Florestal de Linhares, pelo auxílio nos trabalhos de campo; ao Dr. R.M. de Jesús, pelas facilidades concedidas na referida Reserva; ao Dr. A.Gentry pela leitura do manuscrito e sugestões.

\section{Referências bibliográficas}

Aguirre, A. 1950. Sooretama - Estudo sobre o parque de reserva, refúgio e criação de animais silvestres Sooretama, no município de Linhares, estado do Espírito Santo. Bol. Min. Agric. 36(4-6): 1-52. 
Borgonovi, M.N. 1975. Reserva Florestal da Companhia Vale do Rio Doce em Linhares, ES - Uma fonte inesgotável de produtos florestais. Brasil Florestal 6(23): 36-47.

Collar, N.J., Gonzaga, L.A.P., Jones, P.J. \& Scott, D.A. 1987. Avifauna da mata atlântica. Anais do Seminário - Desenvolvimento econômico e impacto ambiental em áreas do trópico úmido brasileiro - a experiência da CVRD: 73-84, Rio de Janeiro.

Davis, T.A.W. \& Richards, P.W. 1933-1934. Britsh Guiana: An ecological study of a limited area on tropical rain forest, part I and II. J. Ecology 21: 350-384; 22: 106-155.

Egler, W. 1954. Rio Doce, enigma do passado, promessa no presente. Bol. Geogr. 1 (7): 42-46.

Grubb, P.J., Lloyd, J.R., Pennington, T.O. \& Whitmore, T.C. 1963. A comparison of montane and lowland rain forest in Equador. I. The forest structure, physiognomy and floristic. J. Ecology 51: 564-599.

Hallé, F., Oldemann, R.A.A. \& Tomlinson, P.B. 1978. Tropical trees and forest. Springer Verlag, Berlin.

Heinsdijk, D., Macedo, J.S. de., Aandel, S. \& Ascoly, R.B. 1965. A floresta do norte do Espírito Santo. Bol. Rec. Nat. Renov. Min. Agric. 7: 1-69.

Jesús, R.M. de. 1982. Produção sustentada em floresta tropical. 1o. Congresso Nacional sobre Essências Nativas, Campos de Jordão. Resumo p. 43.

Jesús, R.M. de. 1987. Mata atlântica de Linhares: Aspectos florestais. Anais do Seminário - Desenvolvimento econômico e impacto ambiental em áreas do trópico úmido brasileiro - a experiência da CVRD: 35-71, Rio de Janeiro.

Köppen, W. 1946. Das geographische system der klimate. In: KOPPEN, W. \& GEIGER, V. ed. Handbuch der klimatologie vol. 1, chap. 3, Teil C. Berlin, Gebr. Borntrager.

Mori, S.A., Boom, B., Carvalho, A.M. \& Santos, T.S. dos. 1983. Ecological importance of Myrtaceae in a eastern brasilian wet forest. Biotropica 15 : 68-70.

Neuwied, M.W. 1820. Viagem ao Brasil nos anos de 1815 a 1817. Ed. Brasiliana, 5a. ser., 1940. Cia ed. Nacional. Rio de Janeiro.

Oldemann, R.A.A. 1974. L'architecture de la forêt guyanaise. Mémoires 73: Paris, OSTROM.

Oldemann, R.A.A. 1989. Dynamics in tropical rain forest. In: Holm-Nielsen, L.B., Nielsen, I.C. \& Baslev, H. ed., Tropical forest, botanical dynamics, spetiation and diversity. Academic Press, New York.

Peixoto, A.L. \& Gentry, A. 1990. Diversidade e composição florística da mata de tabuleiro na Reserva Florestal de Linhares (Espírito Santo, Brasil). Rev. Brasil. Bot. 13:19-25.

Peixoto, A.L. \& Peixoto, O.L. 1986. Nota sobre a ocorrência do Gavião Real (Harpya harpyja) na Reserva Florestal da CVRD. Revista CVRD 7 (26): 56.

Richards, P.W. 1936. Ecological observation on the rain forest of Mout Dulet, Sarawak. J. Ecology 24: 340-360.

Richards, P.W. 1939. Ecological studies on the rain forest of southern Nigeria. The structure and floristic composition of the primary forest. J. Ecology 27: 1-61.

Richards, P.W. 1952. Tropical rain forest - an ecological study. Cambridge Univ. Press. Cambridge.

Rollet, B. 1974. L'architecture des forêts denses humids sempervirents de plaine. Nogent-Sur-Marne: Centre Technique Forestier Tropical - CTFT. 298p.

Saint-Hilaire, A. 1818. Segunda viagem ao interior do Brasil - Espírito Santo. Coleção Brasiliana. vol. 71. Cia. ed. Nacional. Rio de Janeiro.

Schulz, J.P. 1960. Ecological studies on rain forest in northern Suriname. Medded. Bot. Mus. Herb. Rijks Univ. Utrecht 163: 25-32.

Silva, A.F. da. 1980. Composição florística e estrutura de um trecho da mata atlântica de encosta no município de Ubatuba, SP. Tese de Mestrado. Univ. Estadual de Campinas. 153p.

Smith, A.P. 1973. Stratification of temperate and tropical forest. Amer. Nat. 107: 671-683.

Swaine, M.D. 1989. Population dynamics of tree species in tropical forest. In: Holm-Nielsen, L.B., Nielsen, I.C. \& Baslev, H. ed., Tropical forest, botanical dynamics, speciation and diversity. Academic Press, New York.

Withmore, T.C. 1975. Tropical rain forest of the far east. Claredon Press. Oxford. 